\title{
IQRM: real-time adaptive RFI masking for radio transient and pulsar searches
}

DOI:

10.1093/mnras/stab3493

\section{Document Version}

Final published version

Link to publication record in Manchester Research Explorer

\section{Citation for published version (APA):}

Morello, V., Rajwade, K. M., \& Stappers, B. W. (2021). IQRM: real-time adaptive RFI masking for radio transient and pulsar searches. Monthly Notices of the Royal Astronomical Society, 510(1), 1393-1403.

https://doi.org/10.1093/mnras/stab3493

\section{Published in:}

Monthly Notices of the Royal Astronomical Society

\section{Citing this paper}

Please note that where the full-text provided on Manchester Research Explorer is the Author Accepted Manuscript or Proof version this may differ from the final Published version. If citing, it is advised that you check and use the publisher's definitive version.

\section{General rights}

Copyright and moral rights for the publications made accessible in the Research Explorer are retained by the authors and/or other copyright owners and it is a condition of accessing publications that users recognise and abide by the legal requirements associated with these rights.

\section{Takedown policy}

If you believe that this document breaches copyright please refer to the University of Manchester's Takedown Procedures [http://man.ac.uk/04Y6Bo] or contact uml.scholarlycommunications@manchester.ac.uk providing relevant details, so we can investigate your claim.

\section{OPEN ACCESS}




\title{
IQRM: real-time adaptive RFI masking for radio transient and pulsar searches
}

\author{
V. Morello ${ }^{(}, \star$ K. M. Rajwade ${ }^{(0}$ and B. W. Stappers \\ Jodrell Bank Centre for Astrophysics, Department of Physics and Astronomy, The University of Manchester, Manchester M13 9PL, UK
}

Accepted 2021 November 23. Received 2021 November 18; in original form 2021 August 27

\begin{abstract}
In a search for short-time-scale astrophysical transients in time-domain data, radio-frequency interference (RFI) causes both large quantities of false positive candidates and a significant reduction in sensitivity if not correctly mitigated. Here, we propose an algorithm that infers a time-variable frequency channel mask directly from short-duration $(\sim 1 \mathrm{~s})$ data blocks: the method consists of computing a spectral statistic that correlates well with the presence of RFI, and then finding high outliers among the resulting values. For the latter task, we propose an outlier detection algorithm called Inter-Quartile Range Mitigation (IQRM), which is both non-parametric and robust to the presence of a trend in sequential data. The method requires no training and can, in principle, adapt to any telescope and RFI environment; its efficiency is shown on data from both the MeerKAT and Lovell 76-m radio telescopes. IQRM is fast enough to be used in a streaming search and has been integrated into the MeerTRAP real-time transient search pipeline. Open-source PYTHON and $\mathrm{C}++$ implementations are also provided.
\end{abstract}

Key words: methods: data analysis - pulsars: general - fast radio bursts.

\section{INTRODUCTION}

Radio-frequency interference (RFI) broadly refers to all signals that negatively impact radio astronomical observations. While the vast majority emanates from artificial sources, naturally occurring phenomena such as lightning can regularly affect some observatories as well (Sokolowski, Wayth \& Lewis 2015). In radio transient and pulsar searches, idealized data consist of the superposition of a weak astrophysical signal with uncorrelated Gaussian noise in all frequency channels. In this case, the theoretically optimal detection method is known both for individual pulses (Cordes \& McLaughlin 2003) and periodic sources (Morello et al. 2020). Search codes thus generally work under the assumption of a pure Gaussian noise background, which is, however, never realized in practice. The calculation of the detection statistic is dependent on estimating the mean and standard deviation of the background noise from the data; this process is perturbed by the presence of RFI, which can act to reduce the estimated signal-to-noise ratio of a genuine source below the detection threshold. It is necessary to subtract away or mask undesirable signals from the data before they are searched, in order to remain acceptably close to the ideal data assumptions and approach the theoretical sensitivity of the instrument.

RFI mitigation can be performed at all stages of the signal chain, with complementary benefits (see e.g. Baan 2010, for an overview). One can make a broad distinction between pre-detection techniques, which act on the voltage data stream while it is still available at its highest time resolution, and post-detection methods that operate on the channelized, two-dimensional (2D) time-frequency data just before they are searched for radio transients and pulsars. The former

^E-mail: vmorello@gmail.com is better suited to removing short bursts of RFI with minimal data loss; however, they are required to handle large volumes of data in real time and thus tend to be limited to simple thresholding and replacement schemes. A notable exception is that interferometric arrays may implement an additional technique called spatial filtering (e.g. Leshem, van der Veen \& Boonstra 2000), where a null in the beam pattern can be placed in the estimated direction of a strong interference source.

On the other hand, the detection stage involves integrating the baseband data in time, making weaker sources of RFI accessible for removal and reducing the data rate, which in turn enables more sophisticated processing. Post-detection methods usually attempt to blank sections of data across the time and/or frequency dimension based on some specific RFI signatures. Two simple but highly popular methods are the application of a fixed channel mask, which permanently discards the most RFI-occupied portions of the band, and the so-called zero-dispersion measure (DM) filter of Eatough, Keane \& Lyne (2009) that aims to subtract away broad-band signals that show no cold-plasma dispersion expected from propagation through the interstellar medium. An entire class of algorithms involves flagging sections of data based on whether some statistical property exits the range expected for clean data: for example, a rolling sum over multiple time-scales (the SUMTHRESHOLD algorithm of Offringa et al. 2010), or a higher-order statistical moment (Nita \& Gary 2010). Convolutional neural networks have also been trained to perform intelligent clipping of time-frequency data (e.g. Akeret et al. 2017). Fourier transforming the data and finding Fourier bins with excessive power is effective against weaker periodic RFI and well-suited to periodicity searches (Fridman \& Baan 2001; Maan, van Leeuwen \& Vohl 2020).

While there is a trade-off between RFI removal effectiveness and execution speed, the pressure to find methods that perform well on 
both fronts is increasing, and not all the aforementioned methods are fast enough to be used in a streaming environment. Indeed, on the latest and upcoming generations of radio telescopes, an RFI mitigation algorithm should aim to be:

(i) Fast enough to be used on massively multibeam systems, where searching large data streams in real time has now become a fundamental requirement (e.g. CHIME/FRB Collaboration et al. 2018; Rajwade et al. 2021)

(ii) Able to increase the detection probability of genuine sources and reduce the spurious candidate rate at the same time

(iii) Transferable between telescopes with limited adjustments to the algorithm's parameters, which would make it more useful to the community as a whole and avoid duplication of work.

The challenge lies in developing methods that fulfil all three criteria.

Static channel masks may be a blunt tool, but are extremely easy to implement and optimal against portions of the band permanently ridden with RFI. However, the shape and portion of the band occupied by RFI vary as a function of pointing position and of time; a common situation is that a few channels are permanently unusable, while most of them can be occasionally affected with narrow-band RFI; this is arguably the case on MeerKAT, for example (Sihlangu, Oozeer \& Bassett 2020, see also section 3.1). The ability to automatically and accurately adapt a channel mask to the current data on a time-scale of a few seconds is quite desirable; here, we propose an algorithm called Inter-Quartile Range Mitigation (IQRM) that performs this task much faster than real time; IQRM stands for InterQuartile range RFI Mitigation, for reasons that will become clear below.

The outline of the paper is as follows: In Section 2, we explain the rationale behind the design of IQRM before describing the algorithm in detail. In Section 3, we test the ability of IQRM to derive a timevariable channel mask from spectral statistics of data obtained with the MeerKAT and Lovell radio telescopes; its execution speed is also measured. We then evaluate the impact of IQRM in a blind search for single pulses in Section 4; using over $2 \mathrm{~h}$ of known pulsar observations taken with the Lovell Telescope (LT), we compare the number of genuine astrophysical pulses and false positives reported, with and without IQRM applied. We conclude in Section 5 after discussing the performance, limitations and potential extensions to the method.

\section{METHOD}

\subsection{General principle}

Search-mode data is a sequence of dynamic spectra recorded with integration times of typically a few tens to hundreds of microseconds. Given a short-duration block of such time-frequency data, the goal of IQRM is to identify all frequency channels affected by narrowband RFI. The initial step is to reduce the entire block to a single summary statistic per frequency channel (i.e. a spectral moment), and then to determine for which channels the statistic value is unlikely to be associated with clean data. Nita et al. have previously proposed a method where the data are flagged based on their shortterm spectral kurtosis (Nita et al. 2007; Nita \& Gary 2010), for which the acceptable range of values can be determined from first principles. IQRM could be described as a generalisation attempt of the aforementioned work where: (i) Any spectral statistic can be used as the basis to decide which channels are contaminated by RFI, the only requirement being that higher values must indicate a higher probability of contamination.

(ii) The rejection threshold value for the statistic is inferred from the data and assumed to vary slowly with observing frequency; in other words, an arbitrary frequency-dependent background trend or 'baseline' is expected to be present in the sequence of spectral statistic values.

Anything that alters the effective system temperature unequally across the band has the potential to generate such a baseline, for example imperfect bandpass filters, variations in receiver response, the diffuse Galactic radio emission (e.g. Haslam et al. 1981), the presence of a bright radio source with a steep spectral index in the telescope field of view, or broad-band RFI. The fundamental idea of IQRM is to subtract away the baseline problem by taking the so-called lagged differences of the spectral moment values, and then finding high outliers in these difference values, which is a simpler task.

\subsection{The IQRM algorithm}

Formally, the IQRM algorithm takes as its input an ordered sequence of real-valued numbers $\left(x_{i}\right)_{i=0}^{n-1}$ and identifies values significantly larger than their close neighbours in the sequence. $i$ represents a channel index and $x_{i}$ the value of the chosen spectral moment (the measure of RFI contamination) in that channel. IQRM has two adjustable parameters:

(i) The radius $r$ : the distance (expressed in number of sequence elements) to the furthest neighbour being considered when evaluating the outlier status of a given data point in the sequence.

(ii) The threshold $t$ : a significance level (expressed in number of Gaussian sigmas) which controls by how much a data point must exceed one of its neighbours to be categorized as an outlier.

The first step of the algorithm is to calculate a set of lagged differences of the input sequence $\left(x_{i}\right)$. Here, the lagged difference of $\left(x_{i}\right)$ for a given lag $k \in \mathbb{Z}$ is defined as thesequence $\left(\Delta_{i}^{k}\right)_{i=0}^{n-1}$ such that

$\Delta_{i}^{k}=x_{i}-x_{i-k}$,

where the boundary conditions are handled by setting $x_{j}=x_{0}$ for $j<$ 0 and $x_{j}=x_{n-1}$ for $j \geq n$ in the expression above. The usefulness of the lagged difference operation when a trend is present is illustrated in Fig. 1. It is necessary to calculate lagged differences using multiple trial values for $k$ so that a set of consecutive outliers with very similar values does not escape detection. Trial lag values are selected within the range $[-r,+r]$, excluding 0 . To save computation time, we do not try every possible integer value in that range, but instead, arrange the trial values in a geometric progression using the following recurrence relation:

$$
\begin{aligned}
k_{0} & =1 \\
k_{m+1} & =\max \left(\left\lfloor 1.5 \times k_{m}\right\rfloor, k_{m}+1\right),
\end{aligned}
$$

where \lfloor\rfloor denotes the floor function, and noting that all $-k_{m}$ are also added to the sequence of trial values. For example, the sequence corresponding to $r=10$ is $(-9,-6,-4,-3,-2,-1,1,2,3,4,6$, and 9). The geometric progression factor of 1.5 has been chosen as a reasonable compromise between masking accuracy and execution speed, but it has not been subjected to a rigorous optimization process on test data.

Then, for each trial lag value $k$ separately, high outliers in the sequence $\left(\Delta_{i}^{k}\right)_{i=0}^{n-1}$ are then identified using a criterion similar to 

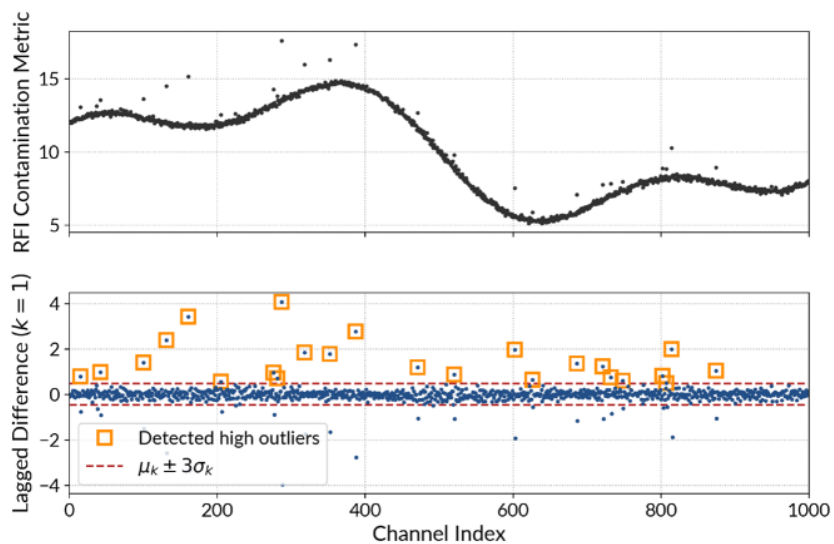

Figure 1. The fundamental idea of IQRM: the lagged difference operation eliminates a slowly varying trend in sequential data and facilitates the procedural identification of outliers. Top panel: artificially generated input data. These are the sum of two sine functions and Gaussian noise; an additional 40 points selected at random were then incremented by an exponentially distributed random variate, to simulate high outliers. Bottom panel: lagged difference of the input data (equation 1) with a lag of $k=1 \mathrm{bin}$; the red dashed lines represent $\mu_{k} \pm 3 \times \sigma_{k}$, i.e. the acceptable range of values empirically inferred using the robust mean and scale estimators of equations (3) and (4). Points highlighted in orange are those deemed abnormally larger than their previous neighbours according to equation (5). IQRM repeats this process for multiple values of the lag $k$ in order to overcome cases where consecutive points are outliers with similar values.

Tukey's rule for outliers, also known as Tukey's fences (Tukey 1977). Here, we assume that the $\Delta_{i}^{k}$ values (for fixed $k$ ) contain:

(i) A majority of inliers that follow some underlying distribution with mean $\mu_{k}$ and standard deviation $\sigma_{k}$.

(ii) A minority of outliers that span a range of values much greater than $\sigma_{k}$

$\mu_{k}$ and $\sigma_{k}$ need to be measured using robust estimators, i.e. that are not easily biased by outliers. We use the following:

$\mu_{k}=Q_{k}(0.5)$,

$\sigma_{k}=\frac{Q_{k}(0.75)-Q_{k}(0.25)}{\Phi^{-1}(0.75)-\Phi^{-1}(0.25)} \approx \frac{\mathrm{IQR}}{1.349}$,

where $Q_{k}(z)$ denotes the $z$-th empirical quantile of the $\Delta_{i}^{k}$, and $\Phi^{-1}$ is the inverse cumulative distribution function of the normal distribution. In other words, the mean of the inliers is taken to be the median of the $\Delta_{i}^{k}$; the standard deviation of the inliers is taken to be proportional to the empirical inter-quartile range IQR $=Q_{k}(0.75)-$ $Q_{k}(0.25)$, where we used the fact that the inter-quartile range of the normal distribution is $\mathrm{IQR}_{\text {norm }}=\Phi^{-1}(0.75)-\Phi^{-1}(0.25) \approx 1.349$. For every pair $(i, k)$, we then test the condition

$\left(\Delta_{i}^{k}-\mu_{k}\right)>t \times \sigma_{k}$,

which if true denotes that $x_{i}$ is abnormally larger than $x_{i-k}$, but on its own, this does not enable discrimination between cases, where $x_{i}$ is a high outlier that should be flagged, and where $x_{i-k}$ is a low outlier that should be ignored. Flagging any $x_{i}$ for which equation (5) is true for at least one trial lag value $k$ would create a pathological case, where the algorithm would mask the entire neighbourhood of every low outlier against any common sense. Another processing step, illustrated on a simple example in Fig. 2, is required to avoid this situation: when equation (5) is true, we state thereafter that $x_{i-k}$ casts a 'vote' against $x_{i}$, which we note $(i-k) \rightarrow i$. Once collected, the full set of votes can be represented as the edges of a directed graph, where the nodes are array indices. A vote $i \rightarrow j$ is considered valid if and only if $i$ has cast strictly less votes in total than $j$ has received. Any data point that receives at least one valid vote is finally marked as an outlier, and the algorithm returns a binary mask with the same size as the input data sequence. The overall effect of IQRM on real data is shown on an example pulse from a known pulsar recorded with MeerKAT at $L$ Band (Fig. 3).

\subsection{Practical implementation and usage}

A minimal open-source implementation of IQRM in the PYTHON language has been made available. ${ }^{1}$ It is minimal in the sense that it provides only the outlier flagging capability specified in Section 2.2. Furthermore, identifying sections of data affected by RFI is not sufficient; this information must then be provided to a search code. The difficulty that arises here comes from the fact that IQRM is meant to generate a channel mask that varies in time: it reads consecutive data blocks of a few seconds in length, calculates their spectral standard deviation (or other contamination statistics of choice), and obtain a mask adapted to each block by applying IQRM. However, with the notable exception of PRESTO (Ransom, Eikenberry \& Middleditch 2002), the dedispersion stages of most widely used search pipelines are not designed to deal with time-variable masks, and instead only accept a fixed list of channels to ignore; such is the case of SIGPROC (Lorimer 2011), HEIMDALl, ${ }^{2}$ PEASOUP, ${ }^{3}$ and ASTROACCELERATE (Adámek \& Armour 2020). In many use cases, it is thus necessary to replace the bad sections of the original data with adequate substitution values, before sending the edited data for processing. For that purpose, we provide a $\mathrm{C}++$ implementation, IQRM APOLLO, ${ }^{4}$ that performs the full sequence of operations: reading, calculation of the spectral moment, flagging, and replacing. It currently processes SIGPROC filterbank files and saves cleaned copies, noting that interfaces to other formats can be added. There are three possible replacement policies for flagged data:

(i) Replace by a constant value of the user's choice.

(ii) Replace by the mean of the medians of the non-flagged channels within the block.

(iii) Replace by artificial Gaussian noise, with mean and variance equal to the global median and global variance of the non-flagged channels within the block.

Mean-of-medians is the default and recommended option, noting that the code is modular and other data replacement strategies can be implemented.

\section{TESTS ON SPECTRAL STATISTIC SAMPLES}

In this section, we test the efficacy of the IQRM masking process described in the previous section on spectral statistic samples obtained from real-world observations.

\subsection{MeerTRAP}

MeerKAT is an array of 64 antennas in the Karoo region of South Africa (Jonas \& MeerKAT Team 2016), each $13.5 \mathrm{~m}$ in diameter and

\footnotetext{
${ }^{1}$ https://github.com/v-morello/iqrm

${ }^{2}$ https://sourceforge.net/projects/heimdall-astro/

${ }^{3}$ https://github.com/ewanbarr/peasoup

${ }^{4}$ https://gitlab.com/kmrajwade/iqrm_apollo
} 

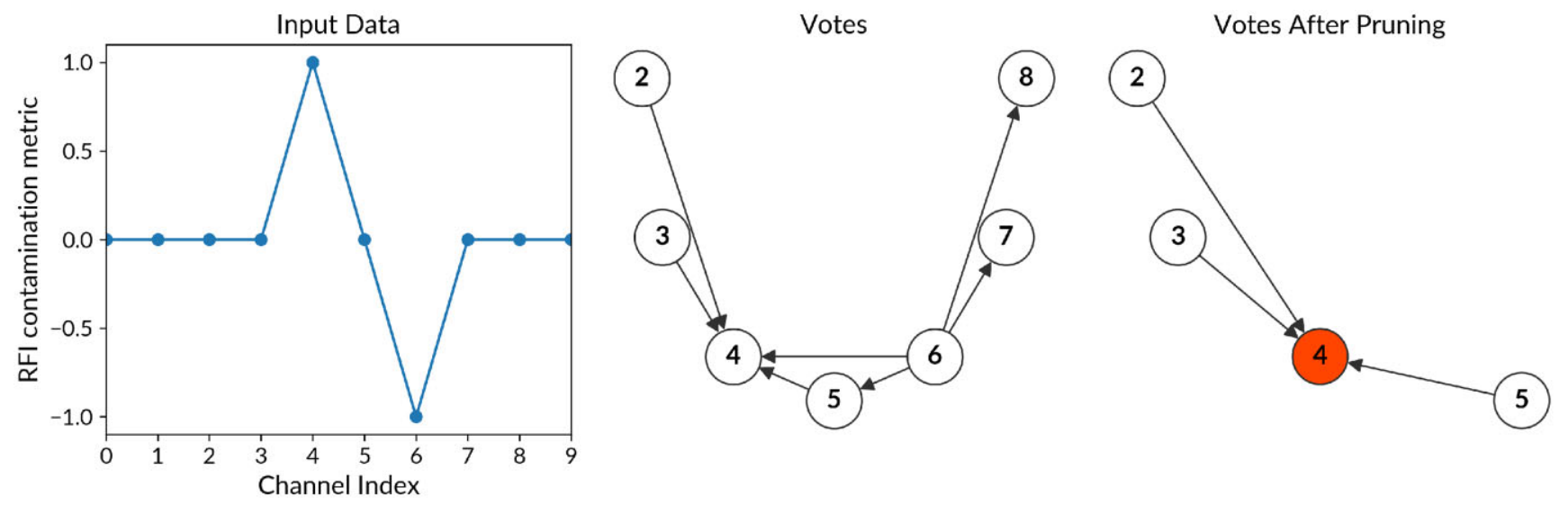

Figure 2. Illustration of the IQRM 'voting' system on a simple test case. Left-hand panel: input data, deliberately generated so that all non-zero lagged differences are considered significant by the algorithm [through equation (5)]. IQRM was then run with a threshold of 3.0 and a radius of 2 channels, which corresponds to trial lag values of $\{-2,-1,+1$, and +2$\}$. Middle panel: summary of votes cast by channel indices on their neighbours, represented as a directed graph. A vote $i \rightarrow j$ is considered valid if and only if $i$ has cast strictly less votes in total than $j$ has received; this ensures that neighbours of low outliers are not abusively flagged. Here, every vote cast by channel 6 is invalid. Right-hand panel: final vote graph after pruning invalid votes; only channel 4 gets marked as a high outlier, reasonably so.
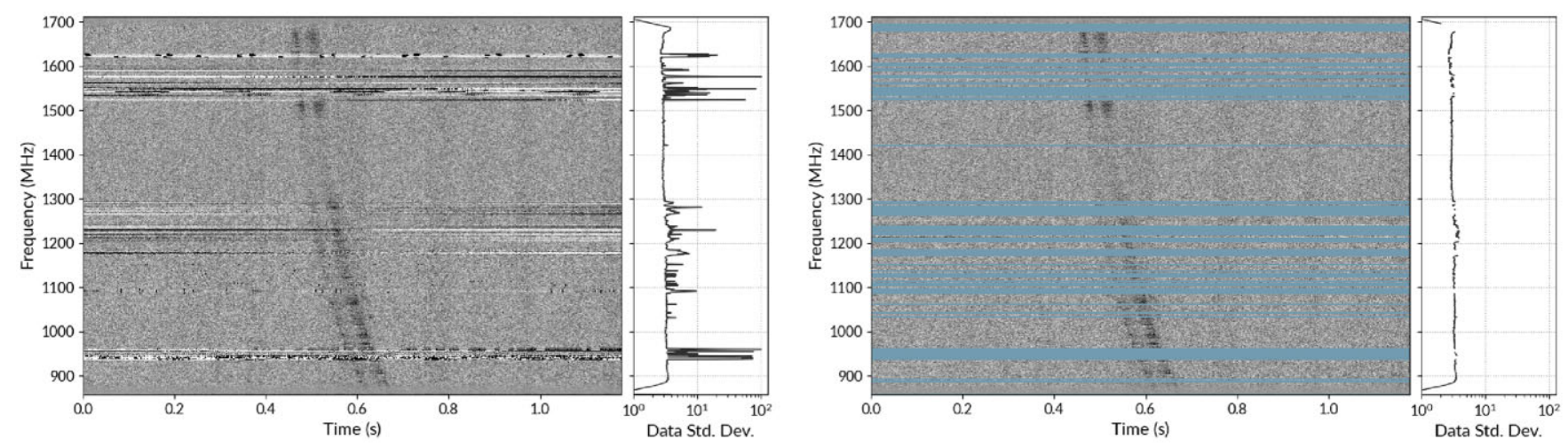

Figure 3. A single pulse from RRAT J1226-3223 recorded with MeerKAT at $L$ Band during the commissioning phase of the MeerTRAP transient search backend (see Section 3.1 for a system description) in July 2019. Left-hand panel: original time-frequency data block; here, the spectral standard deviation of the block was provided as the input to IQRM, where the radius parameter was set to $r=100$ and the threshold to $t=3.0$. Right-hand panel: same data where the frequency channels flagged by IQRM are shown in blue; the colour scale for valid data and the axis ranges are otherwise unchanged. 17 per cent of the channels were masked in this example.

with the capacity to host four different receivers; both an $L$ band (856-1712 MHz) and a UHF (544-1088 MHz) receiver are currently operational. The raw voltage signals are digitized at the antennas and then streamed to the central correlator and beamformer (CBF), which handles channelization followed by correlation and/or beamforming. The $\mathrm{CBF}$ is designed to cover the needs of most telescope users while remaining cost-effective and does not feature a massively multibeam mode for radio transient searches, which greatly benefit from a wide field of view. However, MeerKAT can host additional backends called USEs (for user-supplied equipment) that may receive a copy of the antenna signals via the CBF and implement additional functionality. MeerTRAP is the commensal, real-time radio transient, and pulsar search processor for the MeerKAT radio telescope. It is the association of two sets of user-supplied equipment:

(i) FBFUSE: a GPU-based beamforming cluster capable of tiling the primary field of view of the telescope with over a thousand tiedarray beams (Barr 2018).

(ii) TUSE: a 66-node search cluster that ingests the beamformed data streams from FBFUSE and searches them for radio transients in real time. The raw data are immediately discarded after processing due to their large volume, and only a limited number of data products are kept.

A more detailed system description can be found in Rajwade et al. (2021). The configuration of both components is flexible, but in most observations, they are set so that TUSE searches 768 beams tiled in a hexagonal pattern centred on boresight position, and where the data are digitized to 8-bit precision, contain 1024 frequency channels and have a sampling interval of either $306 \mu$ s (at $L$ band) or $480 \mu$ s (at UHF). In parallel to the search pipeline, each TUSE node runs a socalled bandpass monitor that measures and records spectral statistics of the data for every beam every $6 \mathrm{~s}$, which has provided the test data set for this section. A very important detail to mention here is how the mean and scale of the beamformed data are set on FBFUSE before they are 8-bit digitized. The process is as follows:

(i) A command to initiate a data rescaling is received, which happens when moving to a new source or at the user's request.

(ii) FBFUSE waits until its first input ring buffer block has been filled with data from the CBF. The block has three dimensions: antenna index, observing frequency, and time. It contains approximately $0.3 \mathrm{~s}$ worth of data. 

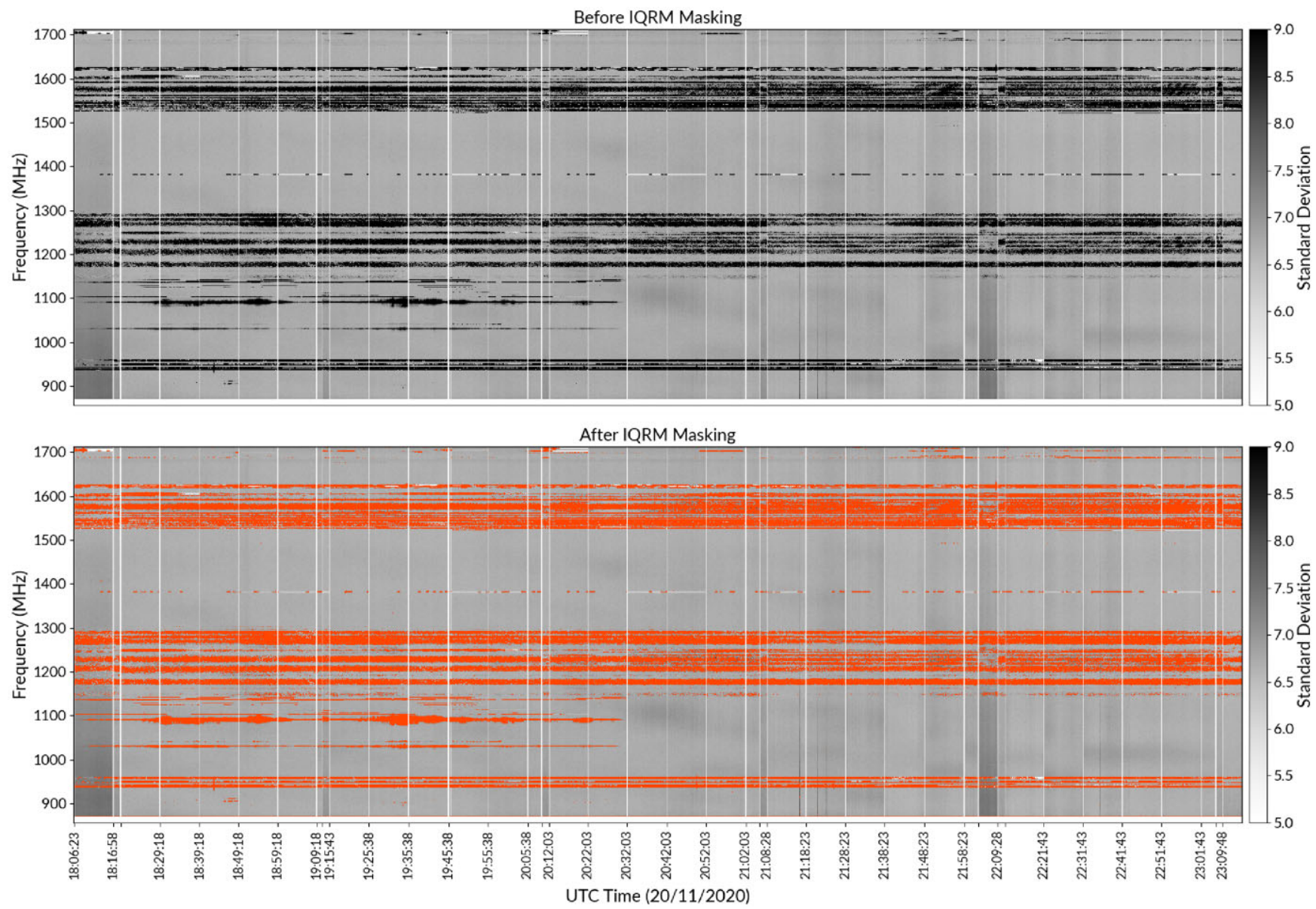

Figure 4. Test of IQRM on $5 \mathrm{~h}$ of MeerTRAP $L$ Band, 1024-channel data with a $306.24 \mu$ s sampling interval. Top panel: spectral standard deviation of the data stream from a single tied-array beam as a function of time, measured on consecutive 6-s data blocks. The beamformer aims to produce 8-bit sampled data scaled to a mean of 127 and a standard deviation of 7.0, which in practice happens only in channels dominated by background noise; higher values are a reliable indicator of RFI (see Section 3.1 for further details). The colour scale has been clipped for readability; some channels can reach a standard deviation $>100$. Vertical lines correspond to short processing interruptions caused by target changes, and adjustments of the beam tiling pattern on the sky that occur every $10 \mathrm{~min}$. Bottom panel: same plot after running IQRM on every spectral standard deviation sample (i.e. every column in the top panel plot), using a radius $r=$ 100 and a threshold $t=3.0$. Channels flagged by IQRM are shown in orange.

(iii) The complex gain corrections are applied to the block.

(iv) The mean and standard deviation of the block are calculated for each channel across all antennas taken together.

(v) Using these estimates, a scale and an offset term are chosen such that the beamformed data has an expected mean of 127 and a standard deviation of 7.0. The scale and offset terms are kept constant and applied to all subsequent blocks until the next rescaling command is received.

The calculation of the spectral scale and offsets assumes that the input data is idealized Gaussian noise: that is, the addition of $\mathrm{N}$ signals with unit scale from $\mathrm{N}$ independent antennas is expected to result in a beamformed output with a scale of $\sqrt{N}$. However, this assumption breaks down in any channel containing highly directional RFI: in these, the signals from distinct antennas retain a certain level of coherence, and the output scale is thus significantly larger than $\sqrt{N}$. Because of this specific data scaling procedure, the spectral standard deviation of the beamformed data received by TUSE has been found to be an excellent indicator of RFI contamination, and can be passed directly to IQRM. The efficacy of IQRM on MeerKAT is shown in Fig. 4, on a 5-h sequence of spectral standard deviation samples acquired at the $L$ band.
We note that a few other spectral statistics were tried in conjunction with IQRM on MeerTRAP data, where the effectiveness of the masking was assessed visually on a sample of known pulsar detections by comparing the data before and after masking as in Fig. 3. These were the absolute values of spectral skewness, the absolute value of spectral excess kurtosis, and the absolute value of the spectral autocorrelation with a 1-sample delay [see Section 3.2 and equation (6) below for a definition]. Absolute values have to be taken for all three to respect the requirement for use with IQRM as stated in Section 2.1, namely that a higher value must indicate a higher probability of contamination. On average, the spectral standard deviation was found to leave fewer contaminated channels unmasked. Rigorously exploring more statistics on a larger sample of data remains to be done.

\subsection{Lovell 76-m radio telescope}

The 76-m LT is located at the Jodrell Bank Observatory in the UK. It lies approximately $25 \mathrm{~km}$ from Manchester's city centre and $15 \mathrm{~km}$ away from its international airport. The RFI environment is thus particularly adverse compared to MeerKAT or any telescope located in a sparsely populated, radio-quiet area. The observing 

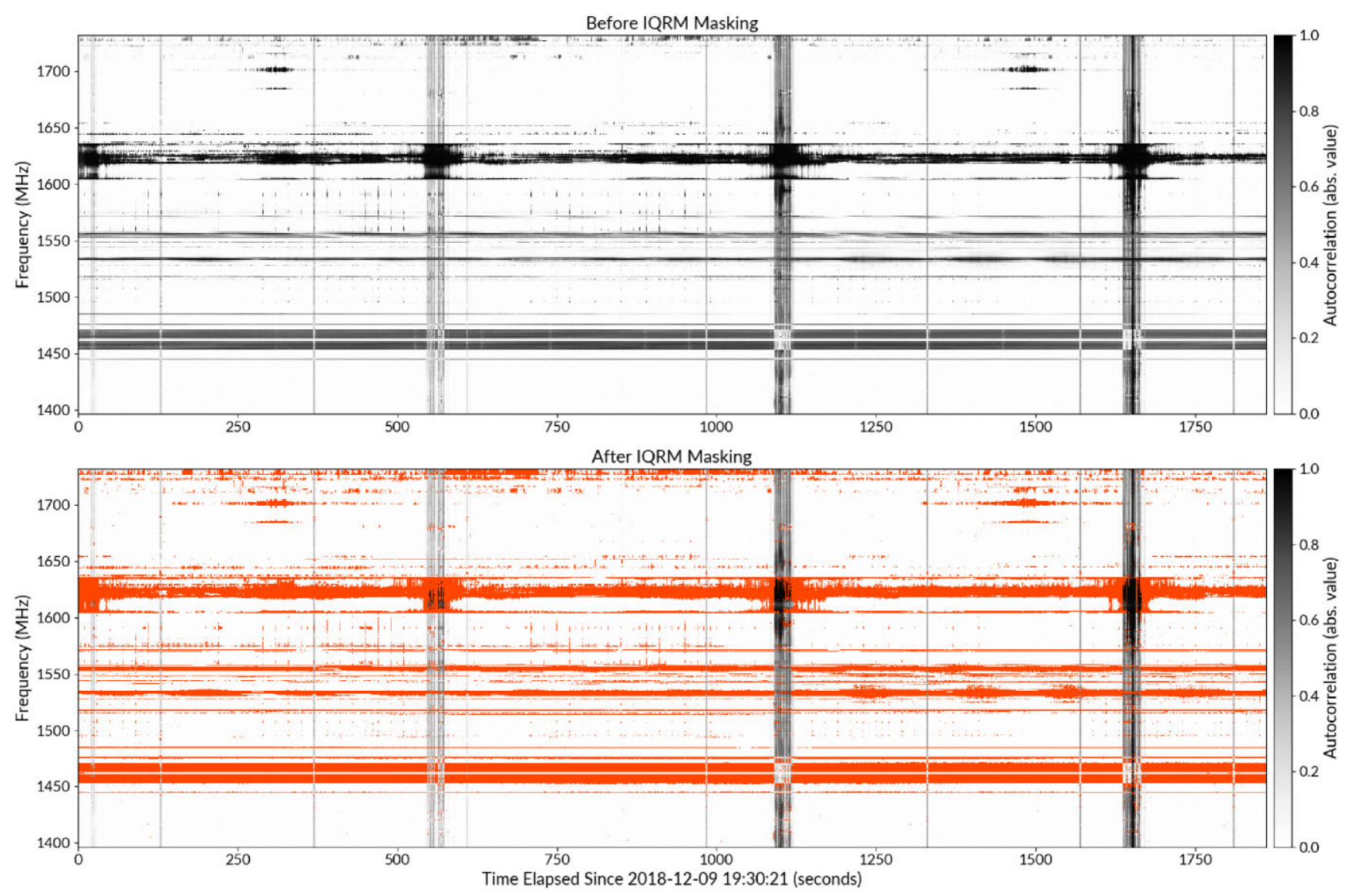

Figure 5. Application of IQRM to a 32-min LT observation of PSR B0611+22, with a $256 \mu$ s sampling interval and 672 frequency channels. On Lovell data, the spectral standard deviation is a much less powerful RFI indicator than on MeerKAT (see Section 3.2 for an explanation), and we have thus selected another spectral statistic: the absolute value of the autocorrelation, with a delay of 1 sample. Top panel: spectral autocorrelation measured on consecutive 0.8 -s blocks. Bottom panel: same data where IQRM has been applied to every column in the top panel; we used a threshold $t=3.0$ and radius $r=67$, i.e. 10 per cent of the number of channels. Flagged channels are shown in orange. A pathological case of the algorithm is visible on a few short time intervals here: it fails when a majority of channels are dominated by RFI.

system has been previously described by Rajwade et al. (2020). The effective observing band spans $336 \mathrm{MHz}$ between 1396 and $1732 \mathrm{MHz}$, divided into 672 frequency channels, and the data are digitized to 8-bit precision. In contrast to MeerTRAP, however, the spectral standard deviation of the data does not correlate well with RFI contamination here; the variance of the data was often found to be smaller in interference-ridden channels. The most plausible explanation is that the low-noise amplifiers of the receiver operate outside of their linear range in the presence of strong RFI, a regime beyond which their output signal power may become a decreasing function of input signal power, contrary to design expectations (Mickaliger, private communication). It was thus necessary to use another spectral moment as an input to IQRM. After testing the same set of spectral statistics that were tried on MeerTRAP data, we found that the best alternative was the absolute value of the spectral autocorrelation with a 1-sample delay, $|\mathrm{ACF} 1|$ hereafter defined as:

$|\mathrm{ACF} 1|=\frac{\left|\sum_{i=0}^{n-2}\left(X_{i+1}-\mu\right)\left(X_{i}-\mu\right)\right|}{n \sigma^{2}}$,

where $X_{i}$ denotes a sequence of $n$ samples within a given frequency channel, and $\mu$ and $\sigma^{2}$ are the empirical mean and variance of the $X_{i}$, respectively. $|\mathrm{ACF} 1|$ takes values in the range $[0,1]$ and has all the required properties to be used as a proxy for RFI contamination with IQRM: (i) Higher values indicate a larger departure from ideal uncorrelated Gaussian noise, for which the expected ACF is 0 regardless of time lag.

(ii) It has a computational cost only marginally larger than the spectral standard deviation, which makes it practical for real-time processing.

(iii) It is independent from the scale of the data, and thus insensitive to changes in spectral standard deviation caused by the occasional level compression issues faced on LT data.

A test on a 32-min sequence of spectral |ACF1| from an observation of PSR B0611+22 can be seen in Fig. 5, and shows that IQRM can be used with different spectral statistics as an input. There are, however, short time periods in LT data during which they become essentially worthless across the whole band, as can be seen, for example around $1100 \mathrm{~s}$ after the start of the test observation. This is a case that IQRM cannot properly handle by design, since a fundamental assumption is that the majority of the frequency channels are reasonably clean. In this case, IQRM tends to recommend leaving most or all of the observing band unmasked, whereas the sensible action would be to discard the data block entirely. 


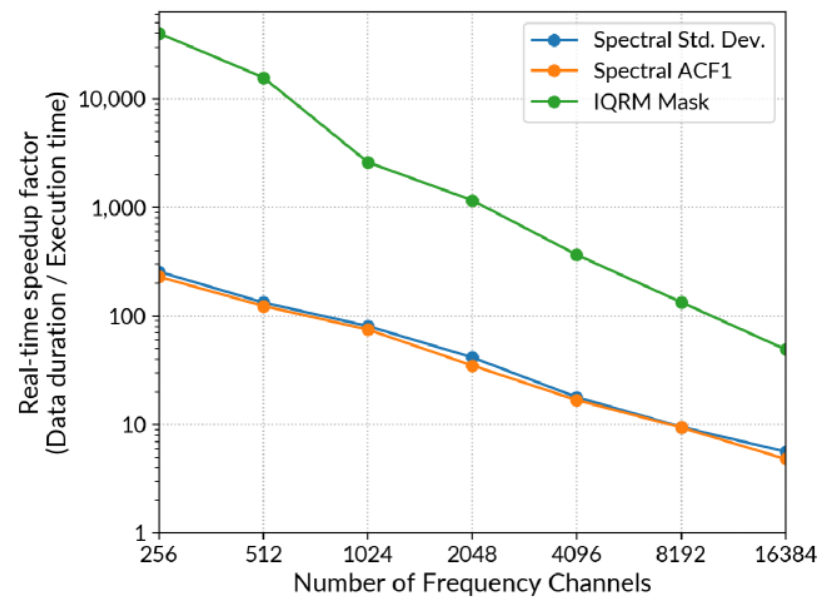

Figure 6. Benchmarks of the IQRM APOLLO implementation. Using a single CPU core, we ran the code on several test data files containing artificial Gaussian noise, with a sampling time $\tau=256 \mu$ s and a number of frequency channels ranging from 128 to 16384 . The data were processed in consecutive blocks of 4096-time samples, approx. $1.05 \mathrm{~s}$ long, and a channel mask calculated for each block. We separately measured the average time consumed by the calculation of the spectral statistic (we tested standard deviation and |ACF1| separately), and by the IQRM masking algorithm per se. The graph shows the ratio between the effective data length and the execution time of each task, i.e. how much faster than real time each task would run in a streaming environment.

\subsection{Execution speed}

We measured the run times of the IQRM APOLLO implementation on search-mode data files with different numbers of frequency channels, all containing artificially generated Gaussian noise. These test files were $60 \mathrm{~s}$ long with a sampling interval of $\tau=256 \mu \mathrm{s}$, and with a number of channels equal to all powers of two between 256 and 16384 inclusive. We set the IQRM parameters to a radius equal to 10 per cent of the number of channels and a threshold of $t=3.0$. The data were processed in blocks of 4096 samples (1.05 s long), and for each block, a channel mask was calculated. We ran the code twice on each file, once using spectral standard deviation as the spectral statistic of choice, and once using the spectral autocorrelation with a lag of 1 sample ( $|\mathrm{ACF} 1|$ as described above). In both cases, we recorded separately the total time taken by the calculation of the spectral statistic and the total time consumed by the IQRM algorithm per se, i.e. the process described in Section 2.2. A single Intel $\mathbb{R}$ Xeon E5-2630 CPU core was used.

The benchmarks results can be seen in Fig. 6; rather than displaying the raw execution times, the ratio between the total data duration and the total run time of each task is shown, which shows how much faster than real time they can be completed in a streaming environment such as MeerTRAP. We note that these results must be linearly extrapolated for a data sampling interval different from $\tau=256 \mu \mathrm{s}$; indeed, the run time-scales linearly with the total number of time samples, but not necessarily with the data duration. On both the MeerTRAP and LT observing systems described above, IQRM can be run approximately 100 times faster than real time on a single core of a recent CPU. It should be noted that the calculation of the spectral statistic dominates the total cost, and in principle, this task could be significantly accelerated using many-core architectures compared to the single CPU core used here. A further speedup of one order of magnitude may be achievable, assuming that computing resources are plentiful enough.
Table 1. Test pulsars chosen for the single-pulse search experiment with the Lovell 76-m telescope at $L$ band. DM denotes the DM and $S_{1400}$ the average flux density at $1400 \mathrm{MHz}$. These sources were chosen so that: a) a large sample of detectable single pulses would be recorded in a short amount of observing time ( $30 \mathrm{~min}$ for each source), and b) the majority of detectable pulses would be near the typical detection threshold of a search, in a regime where the benefits of improved RFI mitigation are most evident. The data in this table are from the ATNF pulsar catalogue (Manchester et al. 2005).

\begin{tabular}{lccc}
\hline Pulsar & Period $(\mathrm{ms})$ & $\mathrm{DM}\left(\mathrm{pc} \mathrm{cm}^{-3}\right)$ & $S_{1400}(\mathrm{mJy})$ \\
\hline PSR B0531+21 & 33.4 & 56.77 & 14 \\
PSR B0611+22 & 334.9 & 96.91 & 3.3 \\
PSR B0919+06 & 430.6 & 27.30 & 10 \\
PSR J1819-1458 & 4263.2 & 196.0 & N/A \\
\hline
\end{tabular}

\section{TESTS ON SINGLE-PULSE SEARCHES WITH THE LOVELL TELESCOPE}

Although the experiments done in the previous section are an important visual check of the IQRM algorithm behaviour on its input, the spectral moment passed to the algorithm constitutes only a proxy for interference contamination. Masking all channels for which the spectral moment of choice is deemed high constitutes no guarantee that RFI has been eliminated. The ground truth, i.e. which exact sections of data are affected by RFI, is never available in practice. The only rigorous way to judge the efficacy of an RFI mitigation method is by measuring how it improves the tangible outcome of the scientific experiment being conducted. In a search for radio transients, one wants to maximize the number of detected pulses from genuine sources while minimizing the number of false positives. In this section, we set out to measure how IQRM improves a standard single-pulse search compared to a fixed channel mask, using observations of known pulsars taken with the LT.

\subsection{Experimental setup}

In order to test the RFI mitigation efficiency of IQRM, we gathered 30-min long search-mode observations of four known pulsars recorded with the Lovell $76-\mathrm{m}$ radio telescope at $1.4 \mathrm{GHz}$, with the goal of submitting them to a single-pulse search. The observing setup was the same as described above (Section 3.2), with a time sampling interval set to $\tau=256 \mu$ s. The test sources were PSR B0611+22, PSR B0919+06, PSR J1819-1458, and PSR B0531+21 (the Crab pulsar), whose parameters are shown in Table 1 . These were selected such that we would be able to detect a large sample of individual pulses within the combined $2 \mathrm{~h}$ of data available, and where a significant fraction of these pulses would be registered with a signalto-noise ratio near the typical detection threshold of a transient search. The processing pipeline consisted of three stages, following the usual processing model for large-scale radio transient searches:

(i) Search. The data were passed to the GPU-based HEIMDALL search code (Barsdell 2012); the signal-to-noise ratio threshold was set to 6 , and the search DM range to $[0,350] \mathrm{pc} \mathrm{cm}^{-3}$. The default internal RFI mitigation options of HEIMDALL were enabled, which consist of narrow-band RFI clipping and a zero-DM filter (Eatough et al. 2009).

(ii) Automated candidate classification. The candidates returned by HEIMDALL were passed to FETCH (Agarwal et al. 2020), a radio transient classifier based on a deep convolutional neural network.

(iii) Manual candidate vetting. The candidates reported as positive by FETCH were visually inspected; the ones confirmed to be originat- 


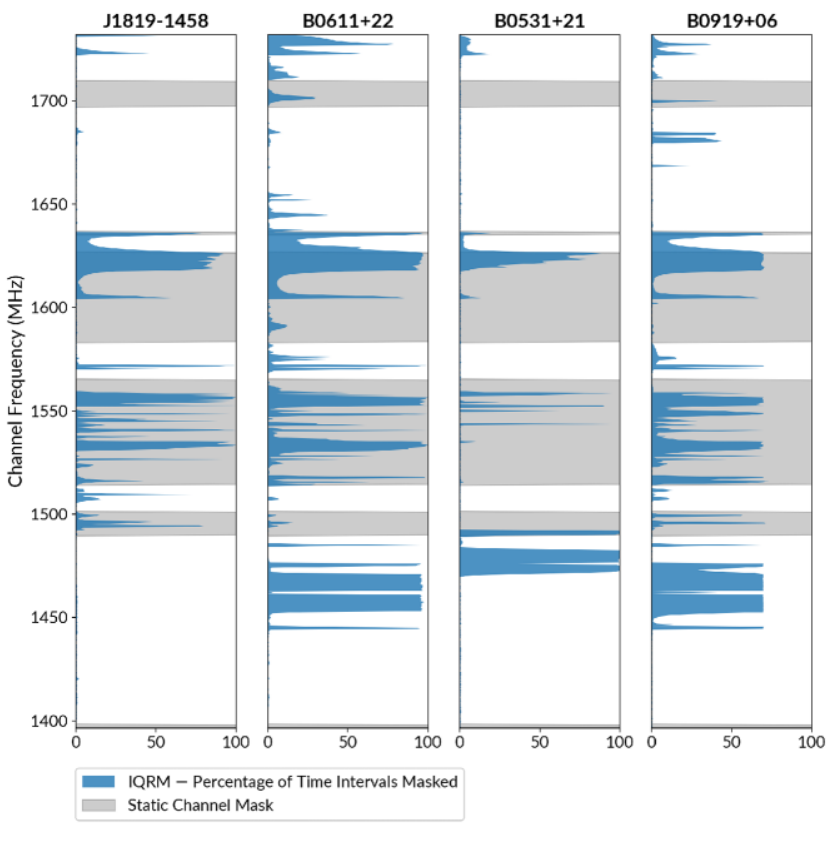

Figure 7. Changes in the RFI environment between test pulsar observations taken with the LT, which underline the need for adaptive time-dependent RFI masking. The percentage of time intervals masked by IQRM as a function of channel frequency is shown in blue. The static channel mask shown in grey is the one commonly used for Lovell $L$ band data and was determined prior to, and independently of this work (see text). Note that many RFI-contaminated channels were saturating near the end of the PSR B0919+06 observation and were thus left unmasked by IQRM, which explains the apparent clipping of the graph.

ing from the pulsar were given a final positive label, the others were overruled as negative.

On each test data file, we ran two identical instances of the pipeline, which processed copies of the data masked with different methods:

(i) One instance processed the original data files with only a static channel mask applied. This mask is the 'standard' for the LT at $1.4 \mathrm{GHz}$ and was determined independently of the present work, mainly via visual inspection of candidates produced by FRB searches conducted with the same backend (Rajwade et al. 2020; Mickaliger et al. (in preparation)]

(ii) The other instance processed copies of the data files that had first been cleaned with the IQRM APOLLO implementation, where we used $|A C F 1|$ as the contamination metric. No static mask was applied.

A comparison of the static channel mask with the average IQRM mask inferred for each test pulsar observation can be found in Fig. 7, where changes of the RFI environment between observations are evident.

\subsection{Results}

The outcome of the search is plotted in detail in Fig. 8, where for each pulsar and channel masking method, the candidates reported as positive by FETCH and confirmed to be genuine pulses by visual inspection are shown in a DM versus time plane as blue circles. Grey crosses denote the candidates labelled as negatives by FETCH, most of which emerge from RFI that was not entirely masked. Table 2 shows the total number of confirmed pulses and negative candidates produced using either RFI mitigation method. Lastly, the confirmed detections from both pipelines were grouped in time and from there it was determined which pulses were detected by both pipelines, and which were exclusively found by either of them. The outcome of this analysis is shown in Fig. 9 as a set of Venn diagrams (one for each test source) and a histogram showing the signal-to-noise ratio distribution of the pulses found only by one of the pipelines.

From the overall number of positive and negative candidates, it is apparent that replacing the commonly used Lovell static channel mask by IQRM is clearly beneficial and that there is no trade-off in doing so, at least in a statistical sense: for every test source, less negative candidates are reported by the search code and, more importantly, a significantly larger number of genuine pulses are found. For all sources combined, there is an overall threefold decrease in the negative candidate rate and a nearly threefold increase in the number of astrophysical events detected. However, IQRM does not achieve a perfect result considering that there is a small number of false negatives, i.e. there are 10 pulses in total that were detected with the static mask but not with IQRM. Out of these 10 events, 3 were labelled as negative by the classifier despite having been found with a marginally higher S/N in the IQRM pipeline, while the other 7 were not detected by the search code. After further inspection of these 7 exclusive detections by the static mask pipeline, 3 were found to have been the product of a chance alignment of the pulse with unmasked narrow-band RFI, which was removed by IQRM. Considering that masking different channels perturbs both the calculation of the detection statistic and the output value of a machine-learning classifier, it is not reasonable to expect a false negative rate of zero even when switching to an RFI mitigation scheme that is clearly superior on average; however, it is reasonable to expect the false negatives to have signal-to-noise ratios close to the detection threshold. Here, all the false negatives are arguably faint, the brightest one having been reported with $\mathrm{S} / \mathrm{N}=8.5$. In contrast, a total of 8 pulses with $\mathrm{S} / \mathrm{N}$ in excess of 15 were missed by the static mask pipeline; all but one are due to mislabeling by the classifier, which was most probably an effect of the residual RFI present in the candidate plots on which it operates.

It is also worth discussing briefly two experimental outcomes that significantly deviate from the average in Table 2. First, the larger than average increase in genuine pulse detections for PSR B0611+22 is worth mentioning. We attribute it to the fact that, during this observation, the vast majority of pulses from the source had signalto-noise ratios (SNRs) concentrated near the threshold of the search code, which was set to 6 . In this regime, the consistent increase in SNR provided by the use of IQRM pushed dozens of pulses just above the detection threshold. Secondly, the exceptionally large decrease of 96 per cent in negative candidates in the PSR B0531+21 observation; closer inspection of the data showed that in this case, most candidates produced in the static mask run are caused by the RFI source occupying the 1450-1470-MHz frequency range, which manifests itself as a square wave with a period usually equal to twice the time sampling interval. This interference source was active in other observations, for example in the PSR B0611+22 one, which can be seen in Fig. 5, where it did not cause a significant portion of the spurious candidates; however, it was unusually bright during the PSR B0531+21 observation, and the fact that the incriminated frequency band is not part of the static channel mask had a significant impact. In contrast, IQRM nearly always masked this portion of the spectrum. 

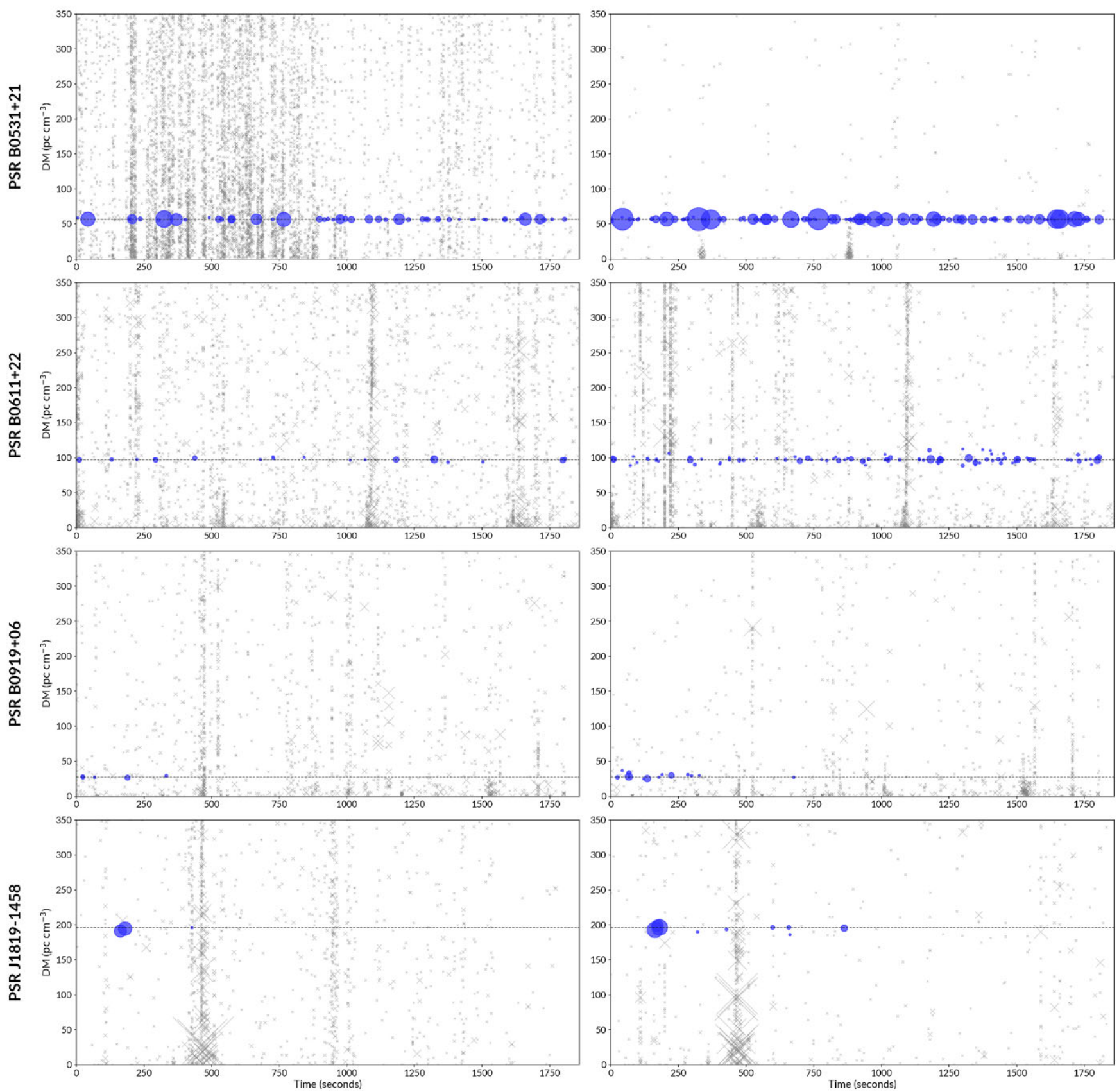

Figure 8. Summary plot of the test single-pulse searches. The four known pulsars in Table 1 were observed for 30 min with the Lovell 76 -m telescope at $L$ band, and the resulting data were searched with two distinct pipelines: one using a static channel mask, and the other using IQRM instead. Both pipelines were otherwise identical. Confirmed pulse detections are shown as blue circles, with the DM of the pulsar shown as horizontal dashed lines. Grey crosses correspond to candidates labelled as negatives by the FETCH classifier. Time is measured in seconds since the start of each 30-min observation. All marker sizes are proportional to the signal-to-noise ratio of the event as reported by the HEIMDALL search code. The total number of pulses and negative candidates for each case is reported in Table 2. Further analysis of which pulses were found by either or both pipelines can be found in Fig. 9.

\section{DISCUSSION AND CONCLUSION}

Implementing effective RFI mitigation is now a necessity on any radio telescope; the task is made even more challenging on recent massively multibeam systems where real-time processing is required. The widely used static channel masks cannot effectively cope with the RFI environment variations as a function of pointing position or time of the day for example. We have introduced IQRM, an algorithm that infers a time-dependent, adaptive channel mask directly from consecutive blocks of search-mode data. Our results show that it fulfils all three desirable characteristics for use in real-time searches with modern massively multibeam systems. First, it runs much faster than real time (approximately 100 times faster on LT data, with a single CPU core), and it should thus be possible to integrate it into any existing data processing pipeline without measurably affecting the speed of the whole search process. Secondly, in a radio transient search, IQRM is able to largely reduce the number of false positives 
Table 2. Summary of candidate numbers produced by the test single-pulse searches. Using IQRM instead of the static channel mask resulted in both a reduction in the number of spurious candidates reported, and in a significantly increased number of astrophysical events identified.

\begin{tabular}{lcccccc}
\hline \multirow{2}{*}{ Source } & \multicolumn{3}{c}{ Confirmed pulse detections } & \multicolumn{3}{c}{ Negative candidates } \\
& Static mask & IQRM & Change & Static mask & IQRM & Change \\
\hline PSR B0531+21 & 51 & 106 & +108 percent & 7079 & 312 & -96 percent \\
PSR B0611+22 & 19 & 95 & +400 percent & 2883 & 2423 & -16 per cent \\
PSR B0919+06 & 5 & 13 & +160 percent & 1442 & 941 & -35 percent \\
PSR J1819-1458 & 3 & 9 & +200 percent & 1134 & 629 & -45 per cent \\
Total & $\mathbf{7 8}$ & $\mathbf{2 2 3}$ & +186 percent & $\mathbf{1 2 5 3 8}$ & $\mathbf{4 3 0 5}$ & -66 per cent \\
\hline
\end{tabular}
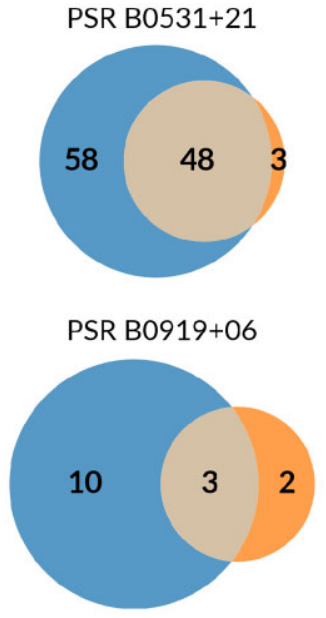
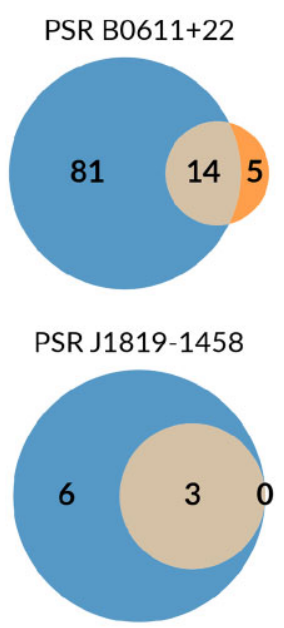

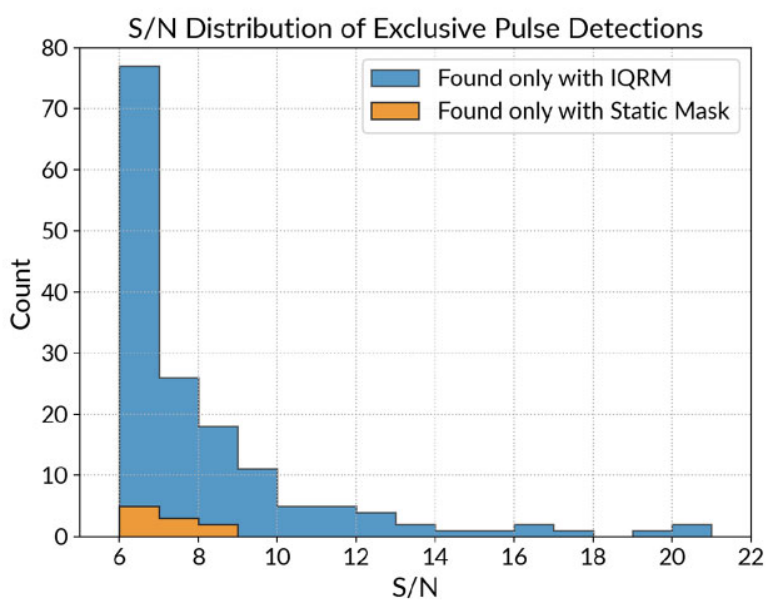

Figure 9. Left-hand panel: Venn diagrams showing the number of confirmed pulses detected by either or both pipelines, for each test source separately. Pulses found exclusively by the IQRM pipeline are shown in blue on the left-hand side of each diagram, while those found only by the static mask pipeline are shown in orange on the right-hand side. The overlapping area in beige represents pulses detected by both. Right-hand panel: reported signal-to-noise ratio distribution of exclusive pulse detections for all sources combined.

but also enhance the $\mathrm{S} / \mathrm{N}$ of genuine astrophysical events; on LT data, the number of individual pulses detected from a set of four known pulsars increased by an average factor of 3 when replacing the standard, commonly used static channel mask by IQRM. Lastly, the algorithm is non-parametric and should thus transfer well between different telescopes. It must be noted, however, that the transfer is not entirely trivial, as one important task is still left to the user: finding a spectral moment or statistic that correlates well with the presence of RFI on the observing system at hand. Our analysis above indicates that the correct choice depends chiefly on the dynamic range of the input data. We found that on MeerKAT, the specific method used to determine the data digitisation levels is such that the standard deviation of the data in a frequency channel is an excellent proxy for RFI contamination, and a natural choice as the IQRM input. On the LT, however, the dynamic range of the data was found to be unexpectedly reduced in the presence of strong RFI bursts, due to a technical limitation of the low-noise amplifiers; this motivated using the spectral autocorrelation of the data as an input to IQRM instead. With that in mind, the IQRM C ++ implementation provides multiple spectral statistics to choose from. The code is modular and users may add new ones if need be.

Much like all existing techniques, IQRM is not universally effective against all forms of RFI and is primarily used as a bright narrowband interference masking algorithm. Its main limitation is that the estimator it uses to infer the acceptable range for its input spectral statistic [equation (5)] implicitly assumes that at least 50 per cent of the channels are clean at any point in time; the method becomes less effective otherwise and masks less data than expected, a situation that was found to occasionally occur on LT data. A possible solution would be to add a short-term memory to the algorithm, i.e. take into account the spectral statistics of past data blocks when determining the acceptable range for said statistic in the most recent block; this could help in making the correct decision when all channels become temporarily contaminated (see Fig. 5). Another potential application of IQRM would be to use the outlier detection algorithm on the data integrated in time, i.e. the zero-DM time series. This could make it useful against broad-band, non-dispersed RFI pulses, much like the now widely used zero-DM filter devised by Eatough et al. (2009), but without systematically reducing the $\mathrm{S} / \mathrm{N}$ of genuine, low DM astrophysical events. The two aforementioned extensions will be considered for a later update of the IQRM APOLLO implementation.

\section{ACKNOWLEDGEMENTS}

VM, KMR, and BWS acknowledge funding from the European Research Council (ERC) under the European Union's Horizon 2020 research and innovation programme (grant agreement No. 694 745). The authors thank Mitchell Mickaliger for useful technical discussions about the LT backend. The authors would like to thank the South African Radio Astronomy Observatory (SARAO) for the approval to use MeerKAT data for the analysis presented in this paper. The MeerKAT telescope is operated by SARAO, which is a facility of the National Research Foundation, an agency of the Department 
of Science and Innovation. Pulsar research at Jodrell Bank and data acquisition with the LT is supported by a consolidated grant from the UK Science and Technology Facilities Council (STFC). We thank the anonymous referee and the editor, Tim Pearson, for their suggestions that helped significantly improve the quality of this article. We also thank Scott Ransom for pointing out an erroneous statement about PRESTO in the initial version of the paper (PRESTO can in fact take a time-variable channel mask as an input, contrary to what was previously claimed).

\section{DATA AVAILABILITY}

The data used in the analysis presented here are publicly available in a repository on Zenodo and can be accessed via a DOI link. ${ }^{5}$

\section{REFERENCES}

Adámek K., Armour W., 2020, ApJS, 247, 56

Agarwal D., Aggarwal K., Burke-Spolaor S., Lorimer D. R., Garver-Daniels N., 2020, MNRAS, 497, 1661

Akeret J., Chang C., Lucchi A., Refregier A., 2017, Astrono. Comput., 18, 35

Baan W. A., 2010, RFI Mitigation in Radio Astronomy. Proc. RFI mitigation workshop — PoS(RFI2010). Sissa Medialab, Trieste, Italy, Available at: https://pos.sissa.it/107/001/pdf

Barr E. D., 2018, in Weltevrede P., Perera B. B. P., Preston L. L., Sanidas S., eds, Pulsar Astrophysics the Next Fifty Years. IAU Symp, Cambridge Univ. Press, Cambridge, UK, p. 175

Barsdell B. R., 2012, PhD thesis, Swinburne Univ. Technology

${ }^{5}$ https://doi.org/10.5281/zenodo.5256885

CHIME/FRB Collaboration et al., 2018, ApJ, 863, 48
Cordes J. M., McLaughlin M. A., 2003, ApJ, 596, 1142

Eatough R. P., Keane E. F., Lyne A. G., 2009, MNRAS, 395, 410

Fridman P. A., Baan W. A., 2001, A\&A, 378, 327

Haslam C. G. T., Klein U., Salter C. J., Stoffel H., Wilson W. E., Cleary M. N., Cooke D. J., Thomasson P., 1981, A\&A, 100, 209

Jonas J., MeerKAT Team, 2016, in Taylor R., Camilo F., Leeuw L., Moodley K., eds, Proc. Sci.. MeerKAT Science: On the Pathway to the SKA, Sissa Medialab, Trieste, Italy, p. 1

Leshem A., van der Veen A.-J., Boonstra A.-J., 2000, ApJS, 131, 355

Lorimer D. R., 2011, Astrophysics Source Code Library, record ascl:1107.016

Maan Y., van Leeuwen J., Vohl D., 2020, A\&A, 650, A80

Manchester R. N., Hobbs G. B., Teoh A., Hobbs M., 2005, AJ, 129, 1993

Morello V., Barr E. D., Stappers B. W., Keane E. F., Lyne A. G., 2020, MNRAS, 497, 4654

Nita G. M., Gary D. E., 2010, MNRAS, 406, L60

Nita G. M., Gary D. E., Liu Z., Hurford G. J., White S. M., 2007, PASP, 119, 805

Offringa A. R., de Bruyn A. G., Biehl M., Zaroubi S., Bernardi G., Pandey V. N., 2010, MNRAS, 405, 155

Rajwade K. et al., 2021, in Evans C. J., Bryant J. J., Motohara K., eds, MeerTRAP in the era of multi-messenger astrophysics. Proc. SPIE 11447, Vol. 11447, Ground-based and Airborne Instrumentation for Astronomy VIII. Available at:https://doi.org/10.1117/12.2559937

Rajwade K. M. et al., 2020, MNRAS, 495, 3551

Ransom S. M., Eikenberry S. S., Middleditch J., 2002, AJ, 124, 1788

Sihlangu I., Oozeer N., Bassett B. A., 2021, Journal of Astronomical Telescopes, Instruments, and Systems, 8,1

Sokolowski M., Wayth R. B., Lewis M., 2015, 2015 IEEE Global Electromagnetic Compatibility Conference (GEMCCON), IEEE, Adelaide, SA, Australia, p. 1

Tukey J. W., 1977, Exploratory Data Analysis. Addison-Wesley, Boston, US

This paper has been typeset from a $\mathrm{T}_{\mathrm{E}} \mathrm{X} / \mathrm{L} \mathrm{T}_{\mathrm{E}} \mathrm{X}$ file prepared by the author. 